\title{
AVALIAÇÃO DE FORNECEDORES DE INSUMOS PARA A CONSTRUÇÃO DE EDIFÍCIOS COM ENFOQUE MULTICRITÉRIO
}

\section{A CONSTRUCTION BUILDING MATERIAL SUPPLIER ASSESSMENT WITH A MULTI- CRITERIA APPROACH}

Recebido em: 28 out. 2019

Aprovado em: 13 fev. 2020

Versão do autor aceita publicada online: 13 fev. 2020

Publicado online: 19 maio 2021

Como citar esse artigo - American Psychological Association (APA):

Gomes, M. M. B., Campos, V. R., \& Chaves, L. G. (2021, jul./set.). Avaliação de fornecedores de insumos para a construção de edifícios com enfoque multicritério. Exacta, 19(3), 678-692. https://doi.org/10.5585/exactaep.2021.15939.

Submeta seu artigo para este periódico $\bigoplus$

Dados Crossmark 


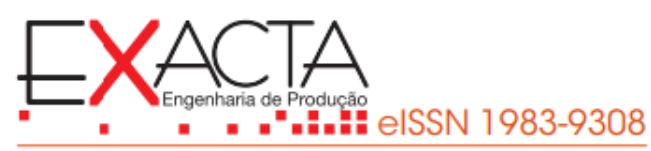

\section{AVALIAÇÃO DE FORNECEDORES DE INSUMOS PARA A CONSTRUÇÃO DE EDIFÍCIOS COM ENFOQUE MULTICRITÉRIO}

\section{A CONSTRUCTION BUILDING MATERIAL SUPPLIER ASSESSMENT WITH A MULTI-CRITERIA APPROACH}

\section{(iD) Maria Micheline Barreto Gomes ${ }^{1}$ \\ iD Vanessa Ribeiro Campos ${ }^{2}$ \\ Dárdner Gadelha Chaves ${ }^{3}$}

\author{
${ }^{1}$ Mestre \\ Universidade Federal do Ceará -UFC. \\ Fortaleza, Ceará - Brasil. \\ michelinebgomes@gmail.com \\ ${ }^{2}$ Doutora \\ Professora da Universidade Federal do Ceará. \\ Fortaleza, Ceará - Brasil. \\ vanessa.campos@ufc.br

\section{${ }^{3}$ Mestre} \\ Universidade Federal do Ceará. \\ Fortaleza, Ceará - Brasil. \\ lardner.chaves@hotmail.com
}

Resumo: A seleção de fornecedores exerce papel significativo no setor de compras de uma empresa de construção civil pois pode reduzir os custos de produção e aumenta a qualidade dos seus serviços ou produtos. Salienta-se que o processo de seleção de fornecedores deve considerar vários critérios, assim, o objetivo da pesquisa é propor uma metodologia para a ordenação de fornecedores de insumos para empresas de construção de edifícios. Foram considerados os insumos mais significativos apontados pela curva $A B C$ conforme uma construtora que atua em Fortaleza e em Juazeiro do Norte no Ceará. A hierarquia obtida, por meio da aplicação do PROMETHEE, considerou como critérios: flexibilidade de pagamento, entrega do produto no prazo, qualidade do produto, assistência técnica. A metodologia proposta permite comparar fornecedores para insumos variados. Pretende-se apoiar as decisões de gestores relacionadas com o suprimento de materiais na construção civil.

Palavras-chave: Gestão de suprimentos. Construção civil. PROMETHEE.

Abstract: Supplier selection plays a significant role in procurement of a construction company because it reduces the production costs and helps increase the quality of services or products. It is noteworthy that the supplier selection process should consider several criteria, therefore the aim of this research is to propose a methodology for suppliers ranking of construction companies. The most significant inputs were pointed out by the material ABC curve designated by a construction company that operates in Fortaleza and Juazeiro do Norte in Ceará. The hierarchy was achieved through the application of PROMETHEE, and the criteria were payment flexibility, delivery on time, product quality, technical assistance. The proposed methodology allows the decision maker to compare various suppliers. This research is intended to support in decisions related to the supply materials for construction.

Keywords: Supply management. Construction. PROMETHEE. 
1 Introdução

A gestão de suprimentos desempenha função estratégica na economia das empresas de construção civil. Ressalta-se que a gestão de suprimentos na construção é responsável por aproximadamente $80 \%$ das atividades de um cronograma para obras de construção civil (Safa, Shahi, Haas, \& Hipel, 2014). Quando as informações dos projetos de construção são insuficientes e os interesses do construtor e dos fornecedores são conflitantes, a seleção de fornecedores se apresenta como atividade desafiadora (Tu, et al., 2017). Nesse contexto, a avaliação de fornecedores possui alto impacto nos diversos setores de uma empresa, tais como: produção, transporte, armazenagem e compras.

A avaliação de empresas fornecimento é um fator crítico de sucesso para as organizações (Aretoulis, Kalfakakou, \& Striagka, 2010; Edyta \& Daniel, 2016). Önüt, Kara, \& Işik (2009) e Zeydan, Çolpan, \& Çobanoğlu (2011) afirma que a escolha de fornecedores realizada com eficácia contribui significativamente para a redução dos custos operacionais, aumenta a competitividade, assegura a qualidade do produto e garante a satisfação do cliente. Escolher fornecedores significa descobrir um profissional que disponibilize um produto de qualidade, nas especificações solicitadas, com preço justo e no prazo acordado (Zhang, Zhang, Lai, \& Lu, 2009).

Em muitas situações, a avaliação de empresas de suprimentos é realizada por meio de apenas um critério como o menor preço (Morkunaite, Podvezko, Zavadskas, \& Bausys, 2019). Pode-se considerar na avaliação apenas as experiências passadas de fornecimento, isso faz com que seja desconsiderado os objetivos organizacionais (Chen, Zhou, \& Zhang, 2011).

Quando a seleção de fornecedor considera um único critério, como por exemplo, o menor preço, o sucesso das atividades de suprimento fica comprometido visto que, nesse caso, têm-se uma análise unidimensional. Ressalta-se que nomear os fornecedores adequados envolve mais do que definir uma de lista de preços, pois esse tipo de decisão depende de vários de fatores de natureza quantitativa e qualitativa (Ho, Xu, \& Dey, 2010). Dessa forma, destaca-se a importância de uma abordagem mais abrangente e multidimensional como qualidade do produto, atendimento ao cliente, prazo de entrega entre outros.

Diante do exposto, o objetivo da pesquisa é propor uma metodologia para ordenação de fornecedores de insumos para a construção de edifícios por meio da metodologia de apoio à decisão multicritério. Os resultados apresentados permitem que os gestores aprimorem o processo de seleção, além de conhecer o desempenho dos fornecedores. Foi aplicado o método PROMETHEE (Preference Ranking Method for Enrichment Evaluation) em uma empresa construtora atuante nas cidades de Fortaleza/CE e Juazeiro do Norte/CE. Essa ordenação permitiu uma análise mais criteriosa na escolha 
além de contribuir para a racionalização e transparência do processo decisório na gestão de suprimentos.

\section{Revisão da literatura}

A metodologia de apoio à decisão multicritério, conhecida como MCDA (Multiple Criteria Decision Aid) é usual na construção civil (Stević, Pamučar, Vasiljević, Stojić, \& Korica, 2017). Diante dos métodos de apoio à decisão, o PROMETHEE - Preference Ranking Method for Enrichment Evaluation é capaz de incorporar a preferência do decisor por meio de uma função de preferência. O PROMETHEE, introduzido na década de 1980, tem a finalidade de fornecer uma solução conforme um grupo discreto de alternativas (Adalı, Işık, \& Kundakcı, 2016).

Encontram-se na literatura diferentes aplicações do PROMETHEE para áreas de conhecimento distintas da engenharia como em recursos hídricos e saneamento, na gestão energética, no planejamento e controle da produção, na logística, entre outros (Behzadian, Kazemzadeh, Albadvi, \& Aghdasi, 2010). Ulengin, Topcu \& Önsel (2001) apontam que esse é um método simples capaz de fornecer a hierarquia entre as alternativas para problemas reais. O PROMETHEE formula-se a partir de duas alternativas denominadas de $a$ e $b$ e um conjunto de critérios (C1, C2,.., Cn). A aplicação do PROMETHEE apresenta as etapas descritas em sequência.

Primeiramente, especificam-se as funções de preferência para cada critério por meio de dados numéricos. Nessa etapa, os critérios são comparados conforme o grau de preferência Pj definido pelo decisor. Os valores de Pj podem variar de 0 a 1, portanto:

- se $\operatorname{Pj}(\mathrm{a}, \mathrm{b})=0$, há indiferença entre as alternativas $a$ e $b$;

- se Pj $(a, b) \approx 0$, obtém-se uma preferência fraca de $a$ sobre $b$;

- Se Pj $(a, b) \approx 1$, têm-se uma preferência forte de $a$ sobre $b$;

- Se Pj $(a, b)=1$, existe preferência estrita de $a$ sobre $b$.

Ressalta-se que a função de preferência Pj pode variar de acordo com o problema. São utilizados para o cálculo seis tipos funções: critério usual, critério com forma de $U$, critério em forma de $V$, critério de nível, critério em forma de V com indiferença e critério Gaussiano (Brans, Vincke, \& Mareschal, 1986). Após o cálculo da função Pj, determina-se os índices de preferência $(\pi)$. Esse índice pode ser obtido pela equação:

$$
\pi(a, b)=\frac{\left[\sum_{j=1}^{n} W_{j} P_{j}(a, b)\right]}{\sum_{j=1}^{n} W_{j}}
$$

onde, Wj representa o peso do critério do termo j. 
Observa-se que Wj pode assumir valores iguais se todos os critérios apresentarem o mesmo grau de importância. Em sequência, calcula-se o fluxo positivo $\left(\phi^{+}\right)$e o fluxo negativo $\left(\phi^{-}\right)$para as alternativas de acordo com as equações:

$\phi^{+}(\mathrm{a})=\sum_{\boldsymbol{b}=1}^{\boldsymbol{m}}(\boldsymbol{a}, \boldsymbol{b})$, para $\mathrm{a} \neq \mathrm{b}$

Eq. 2

$\phi^{-}(\mathrm{a})=\sum_{\boldsymbol{b}=\mathbf{1}}^{\boldsymbol{m}}(\boldsymbol{a}, \boldsymbol{b})$, para $\mathrm{a} \neq \mathrm{b}$

Eq. 3

No PROMETHEE I, a ordem parcial é obtida pela interseção das duas ordenações, definidas pelos fluxos $\phi^{+}$e $\phi$. Caso o fluxo positivo seja maior do que o fluxo negativo, a alternativa é considerada melhor e para calcular o fluxo líquido, utiliza-se a equação:

$\phi(a)=\phi^{+}(a)-\phi^{-}(a)$

Eq. 4

Dessa forma, obtém-se a hierarquia final que consiste na diferença entre os fluxos positivos e negativos, assim se pode constatar a melhor e a pior alternativa.

Na literatura, destaca-se a pesquisa de Araz \& Ozkarahan (2007) que aplica o PROMETHEE para analisar o desempenho geral de fornecedores. Na indústria de cimento, Gupta, Anish \& Bhardwaj (2012) aplicam método para selecionar fornecedores de serviço. Chen, Wang \& Wu (2011) empregam o PROMETHEE no campo da Tecnologia da Informação (TI), criando um modelo com sete critérios e um sistema de decisão em grupo.

Adalı, Işık \& Kundakcı (2016) utilizam a lógica fuzzy juntamente com o PROMETHEE para a seleção de fornecedores e, de maneira similar, Shirinfar \& Haleh (2011) usam a lógica fuzzy juntamente com o PROMETHEE e além dos métodos ANP e TOPSIS, em uma empresa de manufatura. Abordagens análogas com a combinação de lógica fuzzy e PROMETHEE também podem ser encontradas nos trabalhos de Chai, Liu \& Xu (2012); Tavakoli, Tabriz, Farahani \& Rezapour (2013); Senvar, Tuzkaya \& Kahraman (2014) e Mahmoudi, Soheil \& Makui (2015). Ao analisar esses estudos de avaliação de fornecedores, algumas observações merecem destaque.

- O uso de MCDA na avaliação é carente da utilização de critérios que incluem fatores econômicos em conjunto com critérios ambientais (Wang, Zhang, Chong, \& Wang, 2017).

- Os critérios financeiros, de entrega de materiais e de qualidade estão presentes em praticamente na maior parte dos estudos relacionados com esse tema (Stević, Pamučar, Vasiljević, Stojić, \& Korica, 2017).

- A pontualidade de entrega e a qualidade do produto podem ser considerados como os critérios mais importantes nesse tipo de decisão (Kannan \& Tan, 2002). 
Polat, Eray \& Bingol (2017), apontam os critérios mais usados na seleção de fornecedores: custo total do produto, condições de pagamento, lead time, flexibilidade na entrega dos materiais, qualidade, período de garantia, além de conhecimento técnico, reputação, histórico e boa comunicação com os fornecedores. Além desses, pode-se incluir a capacidade tecnológica, compromisso, a credibilidade, a cultura organizacional, o desempenho (Viana \& Alencar, 2012), flexibilidade (Thrulogachantar \& Zailani, 2011) e assistência técnica (Plebankiewicz \& Kubek, 2016).

\section{Dados e método}

A etapa inicial da pesquisa foi a revisão bibliográfica com a finalidade de identificar quais são os critérios mais relevantes na seleção de fornecedores de materiais para a construção civil. Após, foi selecionada uma empresa de médio porte, com aproximadamente 300 funcionários, que atua na construção de edifícios. Essa empresa possui sede em Fortaleza e tem sua filial em Juazeiro do Norte/CE. A companhia é certificada pela ISO 9001- 2008 e PBQP-H (Programa Brasileiro de Qualidade e Produtividade do Habitat).

O setor de compras e o engenheiro de planejamento foram os responsáveis pelos dados de obtidos, para a hierarquização dos fornecedores. O decisor indicado foi o engenheiro de planejamento e possui experiência, na sala técnica, desde 2008. Ele está trabalhando por dois anos na empresa, ele possui experiência na área de orçamento, planejamento e projetos. A experiência do engenheiro, foi importante para definir o grau de importância dos parâmetros propostos. Assim, o entrevistado atribuiu valores para cada da alternativa e critério escolhido.

As alternativas foram definidas de acordo com a lista de fornecedores, em seguida, utilizou-se a curva $A B C$ para listar os insumos de maior importância. A curva $A B C$ é uma técnica de gestão de estoque que classifica os materiais em diferentes categorias e pode ser utilizada em conjunto com diversos métodos de apoio à decisão, tais como: Technique for Order of Preference by Similarity to Ideal Solution (TOPSIS), Redes Neurais Artificiais e Programação Linear Multiobjetivo. Essa técnica emprega o princípio de Pareto para classificar os materiais em categoriais: A (itens muito importantes), B (itens moderadamente importantes) e C (itens que são relativamente sem importância) conforme apontado por Li, Wu, Liu, Fu \& Chen (2019).

Os itens da categoria A são considerados os mais importantes segundo o valor monetário e representam $20 \%$ dos produtos e $80 \%$ do valor monetário. Os itens da categoria B, considerar intermediários, correspondem a 30\% dos produtos. Enquanto os itens da categoria C, são vistos como menos significativos e representam 50\% dos itens (Krajewski, Ritzman, \& Malhotra, 2009).

A curva $A B C$ foi fundamental para delimitar o estudo e aplicar o método multicritério para ordenação dos fornecedores dos insumos mais estratégicos utilizados para a construção de edifícios. A pesquisa limitou-se em analisar apenas os itens da classe A, portanto, foram relacionados 23 insumos 
e, para cada um desses, foram listados até três fornecedores. Consequentemente, obteve-se um total de 38 empresas de fornecimento de materiais. A Figura 1 mostra a lista de materiais e os seus respectivos fornecedores que são apresentados por meio de uma numeração.

Figura 1

Relação dos Materiais versus Fornecedores

\begin{tabular}{|c|c|c|}
\hline Item & Material & Alternativa \\
\hline \multirow{3}{*}{1} & \multirow{3}{*}{ Porcelanato $60 \times 60 \mathrm{~cm}$} & Fornecedor 1 \\
\hline & & Fornecedor 2 \\
\hline & & Fornecedor 3 \\
\hline \multirow{3}{*}{2} & \multirow{3}{*}{ Aço CA-50, Conforme NBR 7480} & Fornecedor 4 \\
\hline & & Fornecedor 5 \\
\hline & & Fornecedor 6 \\
\hline \multirow{2}{*}{3} & \multirow{2}{*}{ Elevador de serviço } & Fornecedor 7 \\
\hline & & Fornecedor 8 \\
\hline \multirow{2}{*}{4} & \multirow{2}{*}{ Elevador social } & Fornecedor 7 \\
\hline & & Fornecedor 8 \\
\hline \multirow{3}{*}{5} & \multirow{3}{*}{$\begin{array}{c}\text { Cerâmica } 7,5 \times 7,5 \mathrm{~cm} \text {, Conforme NBR } \\
13818\end{array}$} & Fornecedor 1 \\
\hline & & Fornecedor 2 \\
\hline & & Fornecedor 3 \\
\hline 6 & Placa de is opor $1000 \times 500 \times 25 \mathrm{~mm}$ & Fornecedor 9 \\
\hline \multirow{2}{*}{7} & \multirow{2}{*}{$\begin{array}{l}\text { Argamassa colante AC III, Conforme } \\
\text { NBR } 14081\end{array}$} & Fornecedor 10 \\
\hline & & Fornecedor 11 \\
\hline \multirow{3}{*}{8} & \multirow{3}{*}{$\begin{array}{l}\text { Cimento portland CP I-Z-32, Conforme } \\
\text { NBR } 11578\end{array}$} & Fornecedor 12 \\
\hline & & Fornecedor 13 \\
\hline & & Fornecedor 14 \\
\hline \multirow{3}{*}{9} & \multirow{3}{*}{$\begin{array}{l}\text { Kit porta pronta abrir, Conforme NBR } \\
115930\end{array}$} & Fornecedor 15 \\
\hline & & Fornecedor 16 \\
\hline & & Fornecedor 17 \\
\hline 10 & Laje volterrana protendida para forro & Fornecedor 18 \\
\hline \multirow{2}{*}{11} & \multirow{2}{*}{ Treliça soldada de aço } & Fornecedor 5 \\
\hline & & Fornecedor 6 \\
\hline \multirow{3}{*}{12} & \multirow{3}{*}{ Aço CA-60, Conforme NBR 7480} & Fornecedor 4 \\
\hline & & Fornecedor 4 \\
\hline & & Fornecedor 6 \\
\hline
\end{tabular}

\begin{tabular}{|c|c|c|}
\hline Item & Material & Alternativa \\
\hline \multirow{3}{*}{13} & \multirow{3}{*}{$\begin{array}{l}\text { Bloco de concreto de vedação, Conforme } \\
\text { NBR } 6136\end{array}$} & Fornecedor 19 \\
\hline & & Fornecedor 20 \\
\hline & & Fornecedor 18 \\
\hline \multirow{3}{*}{14} & \multirow{3}{*}{ Tubo de cobre flexível } & Fornecedor 21 \\
\hline & & Fornecedor 22 \\
\hline & & Fornecedor 23 \\
\hline \multirow{3}{*}{15} & \multirow{3}{*}{$\begin{array}{l}\text { Porcelanato } 59 \times 59 \mathrm{~cm} \text {, Conforme NBR } \\
15463\end{array}$} & Fornecedor 1 \\
\hline & & Fornecedor 2 \\
\hline & & Fornecedor 3 \\
\hline 16 & Telha termoacústica trapezoidal & Fornecedor 24 \\
\hline \multirow{3}{*}{17} & \multirow{3}{*}{ Cuba, Conforme NBR 15097} & Fornecedor 25 \\
\hline & & Fornecedor 26 \\
\hline & & Fornecedor 27 \\
\hline \multirow{3}{*}{18} & \multirow{3}{*}{ Bancada em granito } & Fornecedor 28 \\
\hline & & Fornecedor 29 \\
\hline & & Fornecedor 30 \\
\hline \multirow{3}{*}{19} & \multirow{3}{*}{$\begin{array}{c}\text { Bacia sanitária com caixa acoplada, } \\
\text { Conforme NBR } 15097\end{array}$} & Fornecedor 25 \\
\hline & & Fornecedor 26 \\
\hline & & Fornecedor 27 \\
\hline \multirow{2}{*}{20} & \multirow{2}{*}{$\begin{array}{l}\text { Quadro de distribuição de luz e força } \\
\text { completo }\end{array}$} & Fornecedor 31 \\
\hline & & Fornecedor 32 \\
\hline \multirow{2}{*}{21} & \multirow{2}{*}{ Tela soldada tipo Q } & Fornecedor 5 \\
\hline & & Fornecedor 6 \\
\hline \multirow{3}{*}{22} & \multirow{3}{*}{ Tijolo cerâmico, Conforme NBR 15270} & Fornecedor 33 \\
\hline & & Fornecedor 34 \\
\hline & & Fornecedor 35 \\
\hline \multirow{3}{*}{23} & \multirow{3}{*}{ Rodapé de poliestireno } & Fornecedor 36 \\
\hline & & Fornecedor 37 \\
\hline & & Fornecedor 38 \\
\hline
\end{tabular}

Fonte: Autores.

Após a definição dos critérios e das alternativas, a matriz de decisão foi elaborada com a finalidade de avaliar o desempenho dos fornecedores em cada um dos critérios conforme apresentado no Quadro 1. 


\section{Quadro 1}

Matriz de Decisão

\begin{tabular}{|c|c|c|c|c|c|c|c|}
\hline \multirow[b]{2}{*}{ Material } & \multirow[b]{2}{*}{ Alternativas } & \multicolumn{6}{|c|}{ Critérios } \\
\hline & & $\begin{array}{l}\text { Flexibilidade } \\
\text { de pagamento }\end{array}$ & $\begin{array}{l}\text { Entrega } \\
\text { no prazo }\end{array}$ & $\begin{array}{c}\text { Flexibilidade } \\
\text { na data de } \\
\text { entrega }\end{array}$ & $\begin{array}{c}\text { Flexibilidade } \\
\text { na } \\
\text { quantidade }\end{array}$ & $\begin{array}{l}\text { Qualidade } \\
\text { do produto }\end{array}$ & $\begin{array}{c}\text { Assistência } \\
\text { técnica }\end{array}$ \\
\hline \multirow{2}{*}{ M1 } & F1 & $\ldots$ & $\ldots$ & $\ldots$ & $\ldots$ & $\ldots$ & $\ldots$ \\
\hline & F2 & $\ldots$ & $\ldots$ & $\ldots$ & $\ldots$ & $\ldots$ & $\ldots$ \\
\hline
\end{tabular}

Fonte: Autores.

Foram considerados seis critérios de decisão: flexibilidade de pagamento, entrega no prazo, flexibilidade na data de entrega e na quantidade, qualidade do produto e assistência técnica. A matriz de decisão foi preenchida por meio de entrevista com o engenheiro de planejamento da construtora. $\mathrm{O}$ engenheiro avaliou cada alternativa utilizando uma escala de 1 a 5, obedecendo a seguinte classificação: 1- péssimo; 2- ruim; 3-regular; 4- bom e 5- ótimo. Para expressar a importância dos critérios, o engenheiro atribuiu pesos conforme, esses variando entre 0 e 1 , de modo que a soma foi igual a 1. Quanto maior o peso, maior a relevância atribuída pelo gestor ao critério. A ponderação dos critérios abrangeu os seguintes valores:

- Flexibilidade de pagamento-0,3;

- Entrega no prazo-0,3;

- Flexibilidade na data de entrega-0,05;

- Flexibilidade na quantidade -0,05;

- Qualidade do produto-0,25;

- Assistência técnica-0,05.

Observa-se que, para o decisor, os critérios de flexibilidade de pagamento, de entrega no prazo e de qualidade possuem maiores pesos. O decisor afirmou que a flexibilidade de pagamento se torna diferencial na negociação, especialmente nos casos de necessidade de permuta de materiais, enquanto os critérios de entrega no prazo e qualidade do produto são vistos como fatores críticos de confiabilidade.

\subsection{Aplicação do PROMETHEE ॥}

A aplicação do PROMETHEE II pode ser exemplificada por meio de cinco passos:

- Passo 1: comparar as alternativas e encontrar a amplitude dos desvios;

- Passo 2: selecionar uma função de preferência; 
- Passo 3: calcular o índice de preferência agregada;

- Passo 4: calcular o fluxo de superação, ou seja, a preferência de uma alternativa em relação as demais;

- Passo 5: agregar os fluxos de superação e comparar as pontuações, a fim de classificar as alternativas do maior para o menor valor.

Em cada critério foram associados os limites de preferência $(p)$ e de indiferença (q), cujos valores foram 5 e 0 , respectivamente, para todos os critérios em questão. O objetivo é maximizar todos os critérios. A avaliação de cada alternativa para cada critério pode ser exemplificada conforme mostrada no trabalho de Zhao \& Li (2013).

$\mathrm{Na}$ aplicação do método, inicialmente, para cada critério, foi calculada a diferença de desempenho entre cada par de fornecedores (ai, aj), representada por f(ai) - $f(a j)$. Em seguida, para cada critério, foi atribuída uma das seis funções de preferências definidas por Brans, Vincke, \& Mareschal (1986).

A função de preferência adotada foi critério usual. Essa função assume o valor de 1, se a diferença de desempenho for positiva, e assume o valor de zero, se a diferença for negativa ou nula. As equações (2), (3) e (4) demonstram o cálculo do fluxo positivo, o fluxo negativo e o fluxo líquido. A aplicação do PROMETHEE II possibilitou a ordenação dos 38 fornecedores. Em sequência foi realizada a análise de sensibilidade com a finalidade observar o grau de estabilidade das alternativas por meio de mudanças nos pesos dos critérios.

\section{Resultados e discussões}

A partir dos resultados da matriz de decisão, verifica-se que 20 fornecedores obtiveram um ótimo desempenho no critério qualidade do produto, enquanto apenas 4 fornecedores se destacaram no critério flexibilidade de pagamento, conforme mostrado na Figura 2. Esse resultado demonstra que os fornecedores vêm buscando melhorar a qualidade de seus produtos, porém, é preciso melhorar o quesito de flexibilidade de pagamento. Destaca-se que os critérios de entrega no prazo e qualidade do produto obtiveram maiores pesos, portanto são vistos como mais importantes segundo a preferência do decisor. 
Figura 2

\section{Critérios de Seleção de Fornecedores}

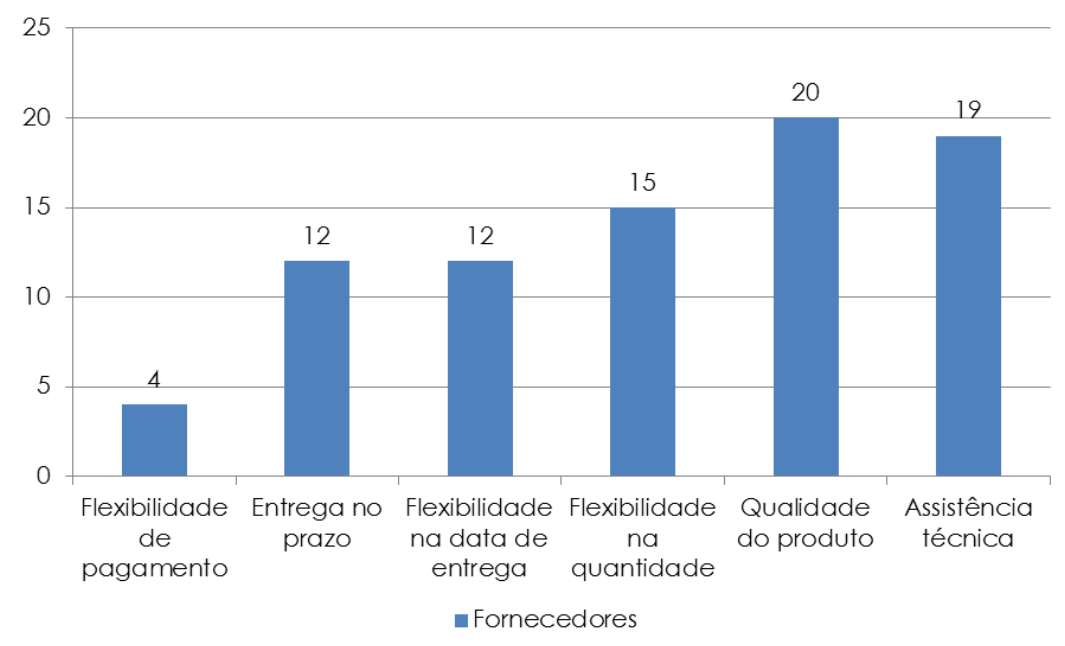

Fonte: Autores.

Após a determinação dos pesos, empregou-se o PROMETHEE II e, finalmente, obteve-se a ordenação dos fornecedores que pode ser visualizado na Figura 3. A ordenação dos fornecedores permitiu conhecer quais fornecedores que apresentam melhor desempenho conforme as preferências do decisor. 
Figura 3

Hierarquia dos Fornecedores por Meio da Aplicação do Método

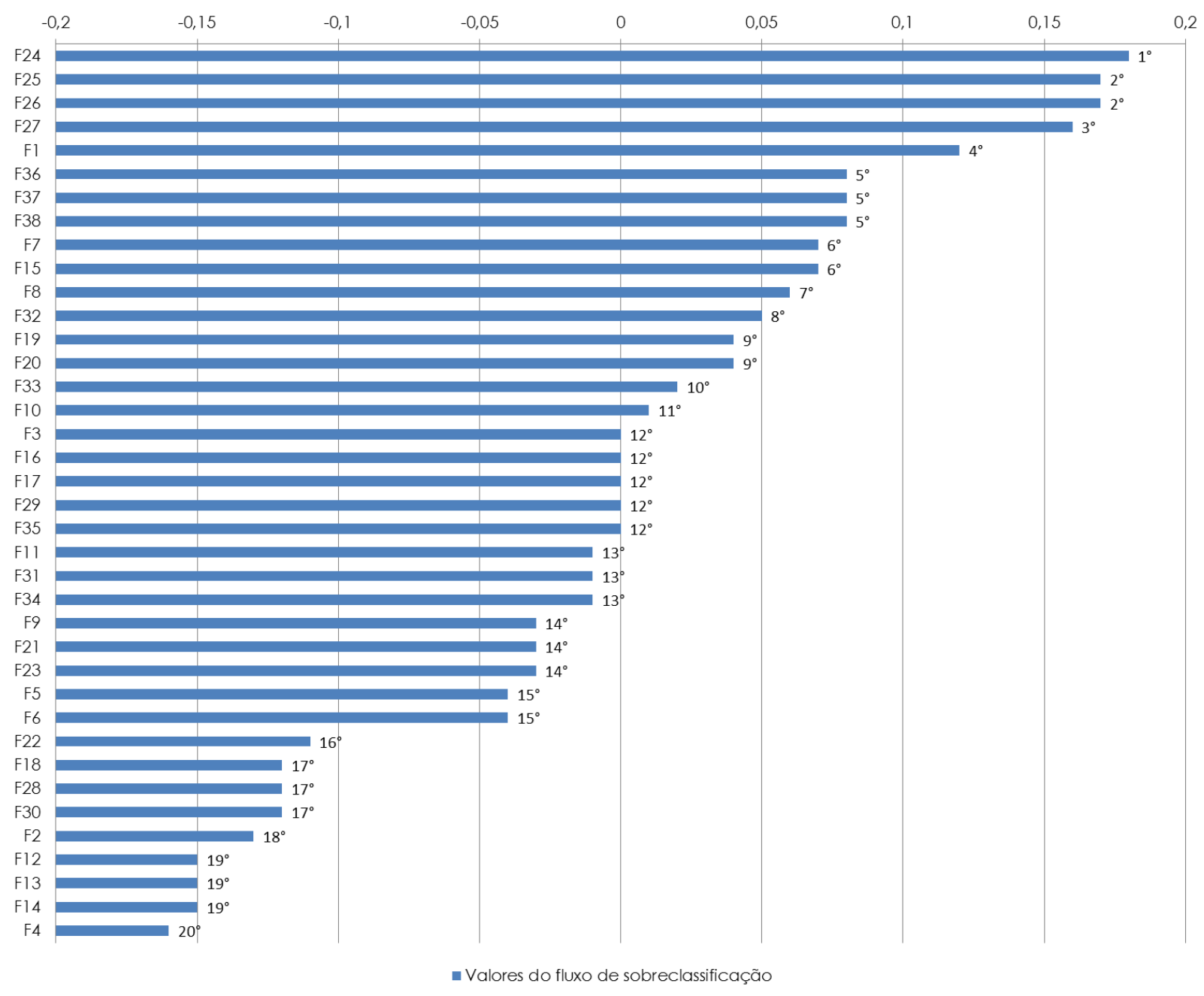

Fonte: Autores.

O resultado indica que os fornecedores F24, F25 e F26 obtiveram as primeiras posições da hierarquia. Ressalta-se que as alternativas F25 e F26 apresentaram o mesmo desempenho. Percebe-se, também, que muitos dos fornecedores obtiveram o mesmo posicionamento na hierarquia. Isso ocorre quando o desempenho dos fornecedores é semelhante para os critérios de decisão considerando a ponderação definida pelo decisor.

A última etapa da aplicação do método foi a análise de sensibilidade, que tem como finalidade estudar o comportamento das alternativas quando se alteram as variáveis do problema. Essa análise apontou que os fornecedores F24, F25 e F26 tiveram bom posicionamento na ordenação conforme indica Figura 4. Isso significa que, mesmo com mudança nas preferências do decisor, essas alternativas apresentam bom desempenho, portanto são consideradas mais estáveis. 
Figura 4

Hierarquia dos Fornecedores por meio da Análise de Sensibilidade

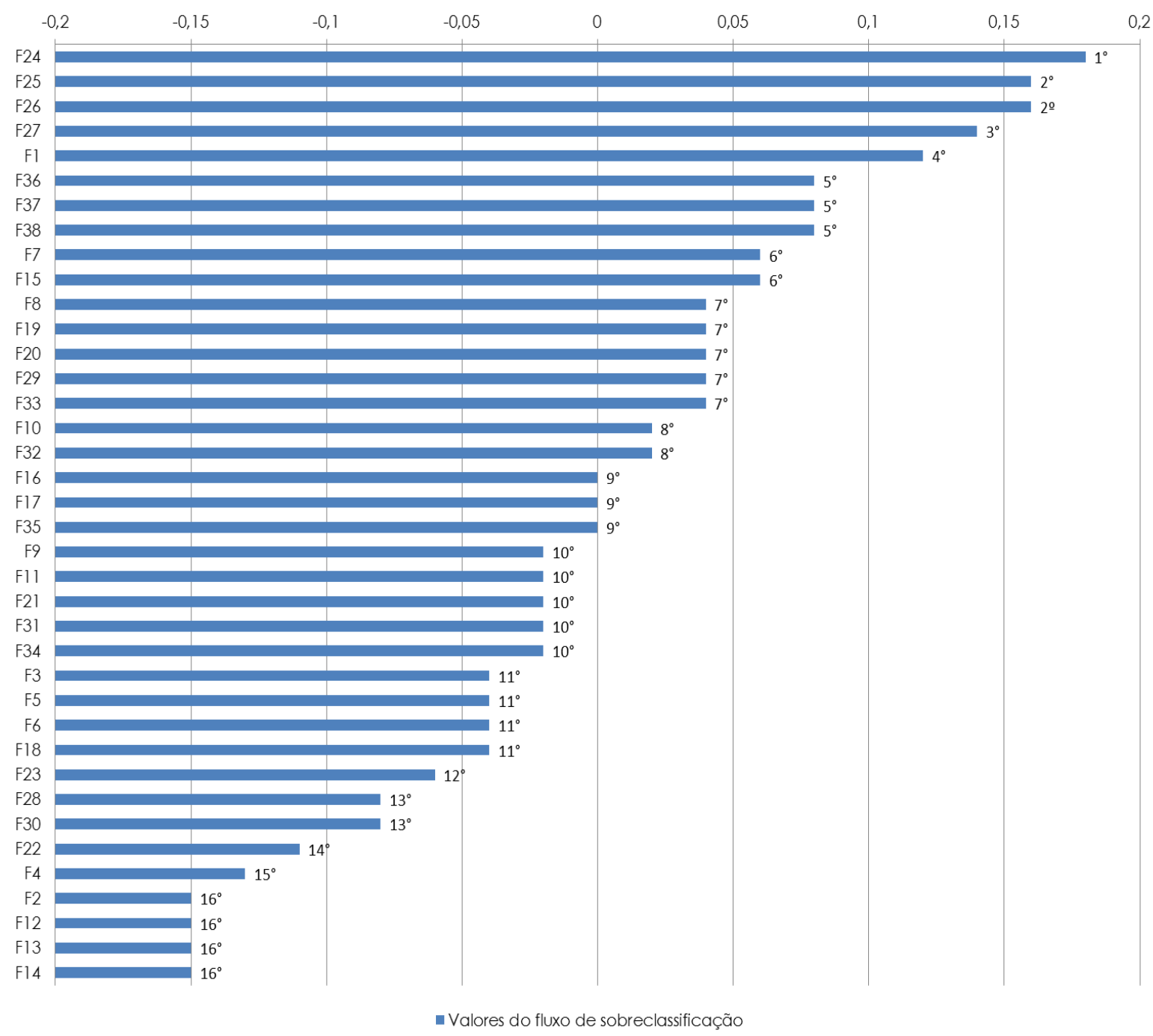

Fonte: Autores.

Foi observado que, ao diminuir o peso dos critérios de flexibilidade de pagamento e de entrega no prazo, ao aumentar o peso dos demais critérios, os seis primeiros colocados mantiveram a mesma ordem e, nas demais posições, não houve uma mudança significativa no resultado. Apesar dessas variações, os resultados foram significativos, evidenciando o impacto dos critérios sobre as alternativas.

De acordo com o método proposto, é possível conhecer o desempenho dos fornecedores, visto que muitas construtoras, em geral, na escolha de fornecedores, analisa-se apenas o custo, não levando em consideração os outros fatores. Nesse caso, o método permite avaliar o desempenho sem considerar o custo do material. Ressalta-se que o custo não foi considerado como critério em função da diversidade dos materiais. No entanto, os critérios considerados são de alta relevância para a avaliação de empresas de fornecimento de insumos de construção. A principal finalidade do método proposto 
não é apenas fornecer uma solução ótima, mas apoiar as empresas de construção civil na gestão de suprimentos.

\section{Considerações finais}

A seleção de fornecedores se constitui como estratégica no desempenho da organização, uma vez que a escolha errônea de fornecedores pode causar problemas financeiros à empresa. Assim, é fundamental que as empresas de construção civil desenvolvam mecanismos que possibilitem trazer melhorias no desempenho dos fornecedores em relação às suas necessidades. A revisão da literatura apontou que a metodologia multicritério de apoio à decisão exerce papel significativo para a gestão de suprimentos de uma empresa.

É importante investir esforços na formulação dos critérios de seleção de fornecedores, pois ,por meio dos resultados obtidos, é possível visualizar a real necessidade da empresa e, consequentemente, cobrar dos fornecedores melhorias. Logo para obter o resultado desejado é necessário tempo e a busca por técnicas de gestão voltadas para apoiar a tomada de decisão.

Esse trabalho, por meio da utilização do PROMETHEE II, obteve como resultado uma hierarquia dos fornecedores, permitindo dessa forma uma análise mais criteriosa na escolha dos fornecedores, e contribuindo para a melhoria da tomada de decisão pelos gestores. Além disso, verifica-se que é possível selecionar fornecedores que reúnam as características necessárias para a construção de parceria.

Observa-se, também, que o desempenho do fornecedor em relação aos critérios adotados influência no resultado final. Outro ponto a destacar nesse estudo é que a qualidade do produto se apresentou como fator primordial e um dos fatores que precisa de melhoria é a flexibilidade de pagamento.

\section{Referências}

Adalı, E., Işık, A., \& Kundakcı, N. (2016). An alternative approach based on fuzzy PROMETHEE method for the supplier selection problem. Uncertain Supply Chain Management, 4(3), 183-194.

Araz, C., \& Ozkarahan, I. (1 de 4 de 2007). Supplier evaluation and management system for strategic sourcing based on a new multicriteria sorting procedure. International Journal of Production Economics, 106(2), 585-606.

Aretoulis, G. N., Kalfakakou, G. P., \& Striagka, F. Z. (2010). Construction material supplier selection under multiple criteria. Operational Research, 10.

Behzadian, M., Kazemzadeh, R., Albadvi, A., \& Aghdasi, M. (1 de 1 de 2010). PROMETHEE: A comprehensive literature review on methodologies and applications. European Journal of Operational Research, 200(1), 198-215. 
Brans, J., Vincke, P., \& Mareschal, B. (1 de 2 de 1986). How to select and how to rank projects: The Promethee method. European Journal of Operational Research, 24(2), 228-238.

Chai, J., Liu, J., \& Xu, Z. (2012). A new rule-based sir approach to supplier selection under intuitionistic fuzzy environments. International Journal of Uncertainty, Fuzziness and Knowledge-Based Systems, 20.

Chen, X., Zhou, X., \& Zhang, Y. (2011). Research on Building Engineering Materials Supplier Selection Based on ANP Method. Advanced Materials and Computer Science. 474, pp. 2089-2094. Trans Tech Publications Ltd.

Chen, Y.-H., Wang, T.-C., \& Wu, C.-Y. (2011). Strategic decisions using the fuzzy PROMETHEE for IS outsourcing. Expert Syst. Appl., 38, 13216-13222.

Edyta, P., \& Daniel, K. (1 de 1 de 2016). Multicriteria Selection of the Building Material Supplier Using AHP and Fuzzy AHP. Journal of Construction Engineering and Management, 142(1), 4015057.

Gupta, R., Anish, S., \& Bhardwaj, A. (2012). Selection of logistic service provider using fuzzy PROMETHEE for a cement industry. Journal of Manufacturing Technology Management, 23, 899-921.

Ho, W., Xu, X., \& Dey, P. (1 de 4 de 2010). Multi-criteria decision making approaches for supplier evaluation and selection: A literature review. European Journal of Operational Research, 202(1), 16-24.

Kannan, V., \& Tan, K. (2002). Supplier Selection and Assessment: Their Impact on Business Performance. Journal of Supply Chain Management, 38(3), 11-21.

Krajewski, L. J., Ritzman, L. P., \& Malhotra, M. K. (2009). Administração de produção e operações (8 ed.). São Paulo: Pearson Prentice Hall.

Li, Z., Wu, X., Liu, F., Fu, Y., \& Chen, K. (2019). Multicriteria ABC inventory classification using acceptability analysis. International Transactions in Operational Research, 26(6), 2494-2507.

Mahmoudi, A., Soheil, S.-N., \& Makui, A. (2015). An extended fuzzy PROMETHEE based on Fuzzy Rule Based System for supplier selection problem. 8.

Morkunaite, Z., Podvezko, V., Zavadskas, E., \& Bausys, R. (1 de 8 de 2019). Contractor selection for renovation of cultural heritage buildings by PROMETHEE method. Archives of Civil and Mechanical Engineering, 19(4), 1056-1071.

Önüt, S., Kara, S., \& Işik, E. (1 de 3 de 2009). Long term supplier selection using a combined fuzzy MCDM approach: A case study for a telecommunication company. Expert Systems with Applications, 36(2), 3887-3895.

Plebankiewicz, E., \& Kubek, D. (1 de 1 de 2016). Multicriteria Selection of the Building Material Supplier Using AHP and Fuzzy AHP. Journal of Construction Engineering and Management, 142(1), 4015057.

Polat, G., Eray, E., \& Bingol, B. (2017). An integrated fuzzy MCGDM approach for supplier selection problem. Journal of Civil Engineering and Management, 23(7), 926-942. 
Safa, M., Shahi, A., Haas, C., \& Hipel, K. (1 de 12 de 2014). Supplier selection process in an integrated construction materials management model. Automation in Construction, 48, 64-73.

Senvar, O., Tuzkaya, G., \& Kahraman, C. (2014). Multi Criteria Supplier Selection Using Fuzzy PROMETHEE Method. Em O. Senvar, G. Tuzkaya, C. Kahraman, C. Kahraman, \& B. Öztay \csi (Eds.), Supply Chain Management Under Fuzziness: Recent Developments and Techniques (pp. 21-34). Berlin, Heidelberg: Springer Berlin Heidelberg.

Shirinfar, M., \& Haleh, H. (2011). Supplier Selection and Evaluation by Fuzzy Multi-Criteria Decision Making Methodology. Int. J. Industr. Eng. Prod. Res, 22, 271-280.

Stević, Ž., Pamučar, D., Vasiljević, M., Stojić, G., \& Korica, S. (2017). Novel Integrated Multi-Criteria Model for Supplier Selection: Case Study Construction Company. Symmetry, 9, 279.

Tavakoli, M., Tabriz, A., Farahani, R., \& Rezapour, E. (2013). Application of Fuzzy Goal Programming \& F-PROMETHEE Approaches in Evaluating and Selecting the Best Suppliers in Supply Chain. Journal of Basic and Applied Scientific Research, 3(2), 1115-1127.

Thrulogachantar, P., \& Zailani, S. (1 de 1 de 2011). The influence of purchasing strategies on manufacturing performance. (Z. Suhaiza, Ed.) Journal of Manufacturing Technology Management, 22(5), 641-663.

Tu, Y., Zhou, X., Gang, J., Xu, J., Shen, W., \& Lev, B. (1 de 9 de 2017). Hierarchical Supplier Selection Optimization with Multiple Items in Large-Scale Construction Projects. Journal of Infrastructure Systems, 23(3).

Ulengin, F., Topcu, I., \& Önsel, Ş. (2001). An integrated decision aid system for Bosphorus watercrossing problem. European Journal of Operational Research, 134, 179-192.

Viana, J., \& Alencar, L. (2012). Metodologias para seleÃßÃ $\mid p o u n d s o$ de fornecedores: uma revisÃ lpoundso da literatura. Production, 22, 625-636.

Wang, T.-K., Zhang, Q., Chong, H.-Y., \& Wang, X. (2017). Integrated Supplier Selection Framework in a Resilient Construction Supply Chain: An Approach via Analytic Hierarchy Process (AHP) and Grey Relational Analysis (GRA). Sustainability, 9, 289.

Zeydan, M., Çolpan, C., \& Çobanoğlu, C. (1 de 3 de 2011). A combined methodology for supplier selection and performance evaluation. Expert Systems with Applications, 38(3), 2741-2751.

Zhang, D., Zhang, J., Lai, K.-K., \& Lu, Y. (1 de 7 de 2009). An novel approach to supplier selection based on vague sets group decision. Expert Systems with Applications, 36(5), 9557-9563.

Zhao, H., \& Li, W. (1 de 1 de 2013). Revised PROMETHEE II for Improving Efficiency in Emergency Response. Procedia Computer Science, 17, 181-188. 